\title{
MOSIC: Mobility-Aware Single-Hop Clustering Scheme for Vehicular Ad hoc Networks on Highways
}

\author{
Amin Ziagham Ahwazi \\ Department of Computer Science and Engineering \\ Islamic Azad University, Ahvaz Branch \\ Ahvaz, Iran
}

\author{
MohammadReza NooriMehr \\ Department of Computer Science and Engineering \\ Islamic Azad University, Ahvaz Branch \\ Ahvaz, Iran
}

\begin{abstract}
As a new branch of Mobile ad hoc networks, Vehicular ad hoc networks (VANETs) have significant attention in academic and industry researches. Because of high dynamic nature of VANET, the topology will be changed frequently and quickly, and this condition is causing some difficulties in maintaining topology of these kinds of networks. Clustering is one of the controlling mechanism that able to grouping vehicles in same categories based upon some predefined metrics such as density, geographical locations, direction and velocity of vehicles. Using of clustering can make network's global topology less dynamic and improve the scalability of it. Many of the VANET clustering algorithms are taken from MANET that has been shown that these algorithms are not suitable for VANET. Hence, in this paper we proposed a new clustering scheme that use Gauss Markov mobility (GMM) model for mobility predication that make vehicle able to prognosticate its mobility relative to its neighbors. The proposed clustering scheme's goal is forming stable clusters by increasing the cluster head lifetime and less cluster head changes number. Simulation results show that the proposed scheme has better performance than existing clustering approach, in terms of cluster head duration, cluster member duration, cluster head changes rate and control overhead.
\end{abstract}

Keywords-Vehicular Ad hoc Networks; Mobile ad hoc Networks; Network Topology Control; Clustering Scheme

\section{INTRODUCTION}

Vehicular ad hoc networks (VANETs) makes a new vision in the field of Intelligent Transportation Systems (ITS). Recently, VANET becomes a most important area of research both in academic and industry field, because it has the potential to create numerous applications such as dissemination of safety, routing plans, traffic condition message, entertainment (e.g. information sharing, gaming), e-commerce and control of vehicle flow formations [1], [21], [22]. In principle, VANET is a special form of MANETs, with the difference that there are mobile nodes (Vehicles) have high dynamic mobility. In VANET vehicles equipped with an on-board unit (OBU) which make theme able to communicate with each other (vehicleto-vehicle, V2V) and via roadside units (vehicle-to-roadside, V2R) also called as RSUs. The communication standard that vehicles used to communicate with each other is Wireless Access for Vehicular Environments (WAVE), which it is an approved amendment to the IEEE 802.11 standard. WAVE is also known as IEEE 802.11p [13].
Due to high mobility, VANET topology changes rapidly, so establishing new control topology cause to introducing high communication overhead for exchanging information. There are several control schemes for media access and topology management have been proposed. One of these schemes is clustering structure. In clustering structure, the mobile nodes are divided into a number of virtual groups based on certain metrics. These virtual groups are called clusters [2].

Some cluster-based approaches have been proposed and applied in Ad-hoc Networks, because the clustering have more advantages such as reduce the delay, overhead and solving the scalability problem in large scale networks. However, in dynamic environments the clusters usually are unstable and frequently disjointed. Hence the clustering schemes which are proposed for Mobile ad hoc networks (MANET) and Wireless Sensor networks (WSN) are not suitable for VANET. On the other word, in VANET, vehicle move with high and variable speeds which causing to frequent changes in the network topology, and it can significantly reduce the cluster stability and efficiency. $\mathrm{CH}$ duration is one of the reasons that can be caused to this reduction. It means that whatever $\mathrm{CH}$ duration increased respectably cluster stability will be increased. On the other hand, an efficient cluster maintenance has directly impact on $\mathrm{CH}$ lifetime. Hence, this parameters should be considered in the design of new cluster scheme. The aim of this work is proposed a scheme to construct a stable single-hop clusters with more $\mathrm{CH}$ lifetime, more $\mathrm{CM}$ duration and less cluster change rate. In this scheme $\mathrm{CH}$ selection conducted base on relative mobility, which calculated as the average relative distance and relative velocity.

The rest of this paper is organized as follows. In Section II previous work related to cluster formation and maintenance will be described. Section III explain preliminaries of proposed scheme. Section IV present our proposed algorithm processing. Section V includes simulation description with comparative results. The paper is concluded in Section VI.

\section{RELATED WORKS}

As a well-known organizing and controlling networks, node clustering is widely used in MANET and Wireless Sensore Networks (WSN). Clustering technique can be used for diverse purpose such as broadcasting, routing and QoS. 
There are many clustering solution based on topology, energy, neighbor have been proposed [16], [7], [8], [9], [10], [11], [12]. However, these clustering algorithms significantly are not suitable for dynamic environments such as VANET. One of the well-known clustering scheme which frequently used for comparison with other VANET clustering algorithms, is MOBIC [4]. Indeed, this algorithm is based on the lowest-ID algorithm [16]. In MOBIC, cluster head selection is based on the signal power which received at any node from its neighbors derived from successive receptions. The performance of MOBIC is medium and not effective for dynamic scenarios.

The aggregate local mobility (ALM) algorithm is proposed in [5]. This algorithm used a relative mobility which calculate based on distance between a node and its neighbors. ALM algorithm aims to extend cluster lifetime using ALM.

Another known clustering algorithm which was proposed is affinity propagation (AP) algorithm [14]. AP algorithm is a distance-based clustering scheme which vehicles exchange the availability and responsibility information with their neighbors and based on this information, $\mathrm{CH}$ is selected. The drawback of AP is that frequent changing of $\mathrm{CH}$ increased when vehicle's speed increased. it is because of that the AP does not take the speed difference of vehicles into consideration.

Adaptable mobility-aware clustering algorithm based on destination positions (AMACAD) [17] is clustering scheme which is proposed for VANETs. This algorithm used set of parameters, including position, speed and distance as a metric for $\mathrm{CH}$ selection. DMMAC is a novel clustering algorithm which proposed by Hafeez et al [15]. DMMAC used velocity as main factor to form clusters, meanwhile it utilized fuzzy system to processing vehicle's velocity to enhance stability of cluster. Beside aforementioned aspects, DMMAC algorithm used a temporary cluster head concept which will be used when the main $\mathrm{CH}$ are unavailable. But this algorithm suffers from $\mathrm{CHs}$ frequently change when the vehicle's speed increased.

At the end of review the previous works, we will refer to laned-based clustering (LBC) scheme [19]. This scheme is designed specifically for the urban environments, which the number of lanes in its traffic flow considered as metric for $\mathrm{CH}$ selection process. However, this scheme does not consider the exact number of vehicles for each flow.

\section{PRELIMINARIES}

The proposed clustering scheme uses Gauss-Markov Mobility (GMM) model [3] to calculate the future vehicles position and based on that predicted position and other metrics (e.g. Relative velocity, relative distance), the proposed scheme try to form a stable single-hop cluster. We call this, MObilityaware and SIngle-hop Clustering scheme (MOSIC). The term of single-hop cluster refer to a cluster architecture which cluster-member can communicate with cluster-head directly. The MOSIC focuses only on V2V (Vehicle-to-Vehicle) communication and the main objective of proposed scheme is to make a large network with highly dynamic nodes appear smaller and able to sustaining clusters for long period by increasing the cluster-head and cluster-member duration.

So in following some essential assumptions and definitions which MOSIC used will be described.

\section{A. Assumptions and Definitions}

The proposed clustering scheme assumes that all vehicles traveling in the same direction (one way) on highway and all of them are equipped with Global Position System (GPS) receivers an On Board Units (OBU). Location information of all vehicles needed for clustering scheme is collected with the help of GPS receivers. Also The roads in highway has a maximum allowed velocity $\left(V_{\max }\right)$. Each vehicle have the same transmitting capability since they have equal chance to be elected as $\mathrm{CHs}$. In this paper we use some definitions that we'll explain them in the following.

In addition, Table II provides the notations that utilize in this paper.

Definition 1: (Vehicle State): In proposed scheme, vehicles have four kinds of state which listed in Table I. Where

TABLE I: Vehicle States

\begin{tabular}{|l|l|}
\hline State & Description \\
\hline$N C$ & Non-Clustered \\
$C M$ & Cluster-Member \\
$C H$ & Cluster-Head \\
$T C M$ & Temporary Cluster-Member \\
\hline
\end{tabular}

$N C$ indicate as a vehicle is standalone and doesn't belong to any cluster, $\mathrm{CM}$ as a vehicle which belong to a cluster, $\mathrm{CH}$ as a vehicle that has task of coordination among cluster members and responsible for management of cluster structure [20] and TCM represent as a vehicle which doesn't receive the information broadcasted by the $\mathrm{CH}$ for $\Delta T$ interval.

Figure 1 show the vehicle's state in a highway environment.

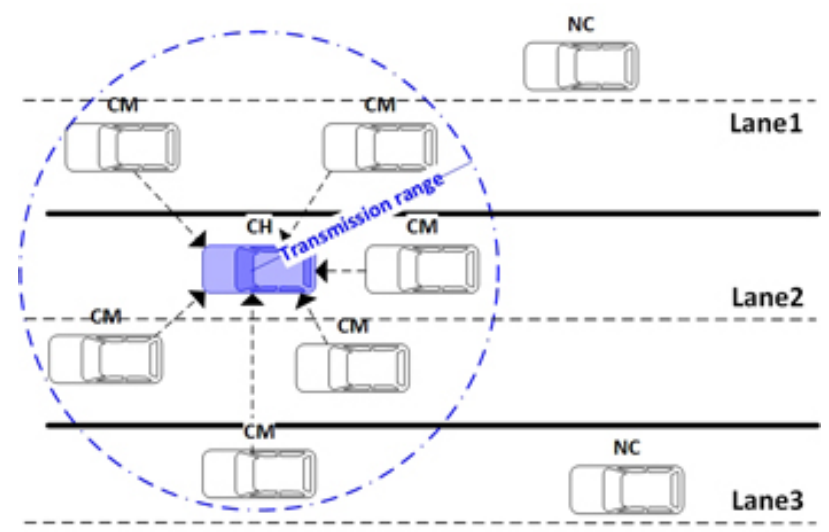

Fig. 1: Vehicle's States

Definition 2: ( $r$-neighbors): We considered two vehicles are $r$-neighbors if the distance between them is less than $r$. Consequently, the neighborhood $N_{i}$ of a vehicle $\mathrm{i}$ is defined as follows:

$$
N_{i}=\left\{j, D_{i, j}<r\right\}
$$

Where $D_{i, j}$ is the average distance between vehicles $i$ and j. 
TABLE II: Notations and Description

\begin{tabular}{|l|l|}
\hline Notation & Description \\
\hline$I D_{i}$ & Unique ID of vehicle $i$ \\
$N_{i}$ & Communication Range of vehicle $i$ \\
$D e g_{i}$ & Neighborhoods of vehicle $i$ \\
$d_{i, j}$ & The nodal degree of vehicle $i$ \\
$d_{i, j}^{\prime}$ & Relative distance between vehicle $i$ and $j$ \\
$x_{i}$ & Predicted relative distance between vehicle $i$ and $j$ \\
$y_{i}$ & Position of vehicle in axe $x$ \\
$x_{i}^{\prime}$ & Position of vehicle in axe $y$ \\
$y_{i}^{\prime}$ & Predicted position of vehicle in axe $x$ \\
$\bar{R}^{\prime}$ & Predicted position of vehicle in axe $y$ \\
$V_{i}$ & Relative Distance \\
$\bar{V}_{m_{i}}$ & Velocity of vehicle $i$ \\
$\bar{V}_{r e l_{i}}$ & Mean relative velocity of vehicle i among their neighbors \\
$V_{m a x}$ & Relative Velocity of vehicle $i$ \\
$\bar{M}_{i}$ & Maximum speed of road \\
$M_{i}$ & Relative Mobility metric of vehicle $i$ \\
$\Delta V_{t h}$ & Mobility metric of vehicle i \\
$C F V$ & The threshold used to classify neighboring nodes as stable or non \\
\hline
\end{tabular}

Definition 3: (Nodal degree): The total number of $r$ neighbors of a vehicle is called the nodal degree of the vehicle $i$ which calculated as follow:

$$
\operatorname{Deg}_{i}=\left|N_{i}\right|
$$

The nodal degree of a node $i$ can be concluded as the cardinality of the set $N_{i}$.

Definition 4: (Stable $r$-neighbors): Two vehicles are considered as a stable $r$-neighbors if the difference speed between them is less than $\pm \Delta V_{t h}$. Where $\Delta V_{t h}$ is a predefined threshold.

\section{B. Gauss-Markov Mobility (GMM) Model}

The Gauss-Markov Mobility (GMM) Model [3] is a memory-based mobility model which able to calculate next position of mobile node based on its current mobility metric. In this model, each mobile node is assigned to the initial speed and direction. The GMM model used alpha $\alpha, 0 \leq \alpha \leq$ 1 , parameter which determines variability in mobile node movement. In this model, at each fixed interval of time, $n$, the mobile node update it current speed and direction which the new speed and direction are calculated as follows:

$$
\begin{aligned}
& s_{n}=\alpha s_{n-1}+(1-\alpha) \bar{s}+\sqrt{(1-\alpha)^{2}} s_{x_{n-1}} \\
& d_{n}=\alpha d_{n-1}+(1-\alpha) \bar{d}+\sqrt{(1-\alpha)^{2}} d_{x_{n-1}}
\end{aligned}
$$

where $s_{n}$ and $d_{n}$ are the new speed and direction of the mobile node at time interval $n ; \bar{s}$ and $\bar{d}$ are representing the mean value of speed and direction and $s_{x_{n-1}}$ and $d_{x_{n-1}}$ are random variables from a Gaussian (normal) distribution. At each time interval the next location is calculated based on the current location, speed, and direction of movement.
Specifically, at time interval $n$, an Mobile node's position is given by the equations 5 and 6 :

$$
\begin{aligned}
& x_{n}=x_{n-1}+s_{n} \cos \left(d_{n-1}\right) \\
& y_{n}=y_{n-1}+s_{n} \sin \left(d_{n-1}\right)
\end{aligned}
$$

where $\left(x_{n}, y_{n}\right)$ and $\left(x_{n-1}, y_{n-1}\right)$ are the $\mathrm{x}$ and $\mathrm{y}$ coordinates of the mobile node's position at the $\left(n^{\text {th }}\right)$ and $(n-1)^{\text {st }}$ time intervals, respectively, and $s_{n}$ and $d_{n}$ are the speed and direction of the mobile node, at the $(n)^{\text {st }}$ time interval which achieved based on equations 3,4 .

\section{Message passing format}

As previously mentioned, the VANET is running under WAVE (Wireless Access for Vehicular Environments) architecture (IEEE 802.11p) and messages are encapsulated in UDP packets in the network layer. Each vehicles exchange their status message with their neighbors in its communication range, $r$, periodically. The status message contains information about the vehicle's ID, vehicle state, current speed $V$, communication range $r$, CH's ID (CHID) and position POS, as shown in Fig. 2.

\begin{tabular}{|l|l|l|l|l|l|}
\hline$I D$ & State & $V$ & $r$ & CHID & POS \\
\hline
\end{tabular}

Fig. 2: Status message packet format

In addition, $P O S$ consist two parts, Geographical and Predicted position, which both of them are based on Cartesian coordinates. The geographical location include $(x, y)$ and predicted location consist $\left(x^{\prime}, y^{\prime}\right)$.

\section{Cluster Metrics}

In this section the cluster metrics, which plays an important role in cluster formation and cluster maintenance, will be described.

1) Average relative velocity: In every time interval, each vehicle will be aware about all $r$-neighbor vehicles, using exchange status message, and based on that information, average relative velocity $\bar{V}_{r e l_{i}}$ will calculated as follow:

$$
\bar{V}_{\text {rel }_{i}}=\max \left\{\frac{\bar{V}_{m_{i}}}{V_{\max }}, 0\right\}
$$

where $V_{\max }$ is the maximum allowed velocity on the road, and $\bar{V}_{m_{i}}$ is the average velocity of vehicle $i$ against their $r$ neighbors which defined as follow:

$$
\bar{V}_{m_{i}}=\frac{1}{\left|D e g_{i}\right|} \sum_{j=1}^{D e g_{i}}\left(V_{i}-V_{j}\right)
$$

where $j$ is a potential neighboring vehicle, and $V_{i}, V_{j}$ are the velocity of vehicle $i$ and $j$ respectively in $\mathrm{m} / \mathrm{s}$ and $D e g_{i}$ is nodal degree of vehicle $i$. 
2) Average relative distance: Each vehicle will collect its mobility information such as its location at every time interval $\Delta T$ and send this information to all its $r$-neighbors via Control Channel. So each vehicle able to calculate its average relative distance among its $r$-neighbors. Relative distance is one of the measure that play a key role to elect $\mathrm{CH}$.

Consequently relative distance defined and calculated as follow:

$$
\bar{R}_{i}=\frac{1}{\left|D e g_{i}\right|} \sum_{j=1}^{\operatorname{Deg}_{i}} R_{i, j}
$$

where $R_{i, j}$ is obtained from the below equation:

$$
R_{i, j}=10 \times \log _{10}\left(\frac{d_{i, j}}{d_{i, j}^{\prime}}\right)
$$

We use the metric proposed by Basu [4] to calculate average relative distance (Equation 10), but whit difference that we used distance and predicted distance between two nodes instead of Packet Delay, which is used in [4]. In formula 10, $d_{i, j}$ is distance between vehicle $i$ and $j$ which can achieved and calculate via Euclidean distance.

$$
d_{i, j}=\sqrt{\left(x_{i}-x_{j}\right)^{2}+\left(y_{i}-y_{j}\right)^{2}}
$$

also $d_{i, j}^{\prime}$ represents the distance between vehicle $i$ and $j$ which is predicted with mobility model, and similar to $d_{i, j}$ calculated as follow:

$$
d_{i, j}^{\prime}=\sqrt{\left(x_{i}^{\prime}-x_{j}^{\prime}\right)^{2}+\left(y_{i}^{\prime}-y_{j}^{\prime}\right)^{2}}
$$

where $x_{i}^{\prime}$ and $y_{i}^{\prime}$ is future position of vehicle $i$ that calculated and obtained using Gauss-Markov Mobility model (see Sect. III-B).

3) Average relative mobility: Average relative mobility is an important measurement that vehicles can be informed about their $r$-neighbors and based on this parameter, vehicles decides which vehicle is more suitable to selected as $\mathrm{CH} . \bar{M}_{i}$ is defined as follow:

$$
\bar{M}_{i}=\bar{R}_{i}+\bar{V}_{\text {rel }_{i}}
$$

where $\bar{R}_{i}$ is average relative distance and $\bar{V}_{\text {rel }}$ is average relative velocity which described in previous subsections.

As you can see, whatever the nodal degree of a vehicle is increased, then correspondingly, the value of $\bar{R}_{i}$ and $\bar{V}_{r e l_{i}}$, will be decreased. Because according to Formula 8 and 9 , the relative distance and relative velocity are inversely proportional with nodal degree $\left(D e g_{i}\right)$. So a vehicle with lower value of $\bar{M}_{i}$ is more considerable.

\section{MOSIC PROCESSING}

This section contains the description of the procedures that form part of the proposed clustering scheme. In brief, the proposed clustering scheme is formed by the four phase (Initialization, CH Selection, Cluster Formation and Cluster maintenance), described in the following subsections.

When a node is not belong to any cluster (in Non-Clustered state), it executes the initialization phase. after that, depending on whether the cluster head can be found in nearby or not, the node can launches the join procedure or the cluster formation phase. Hence, after the cluster formation phase or after joining a cluster, the maintenance procedure will be executed and it checks the validity of the cluster periodically.

\section{A. Initialization phase}

This phase is executed by any vehicle which its state is NC (Non_Clustered) and also receives a status message from its $r$-neighbors. In any interval time, $\Delta T$, a vehicle which its state is NC broadcast its status message to discover weather a Cluster Head exist in vicinity or not. If there is at least one Cluster Head can be found, then the vehicle launches the join procedure. Otherwise, it execute the cluster formation phase. The pseudo code of the Initialization phase shown in Algorithm 1.

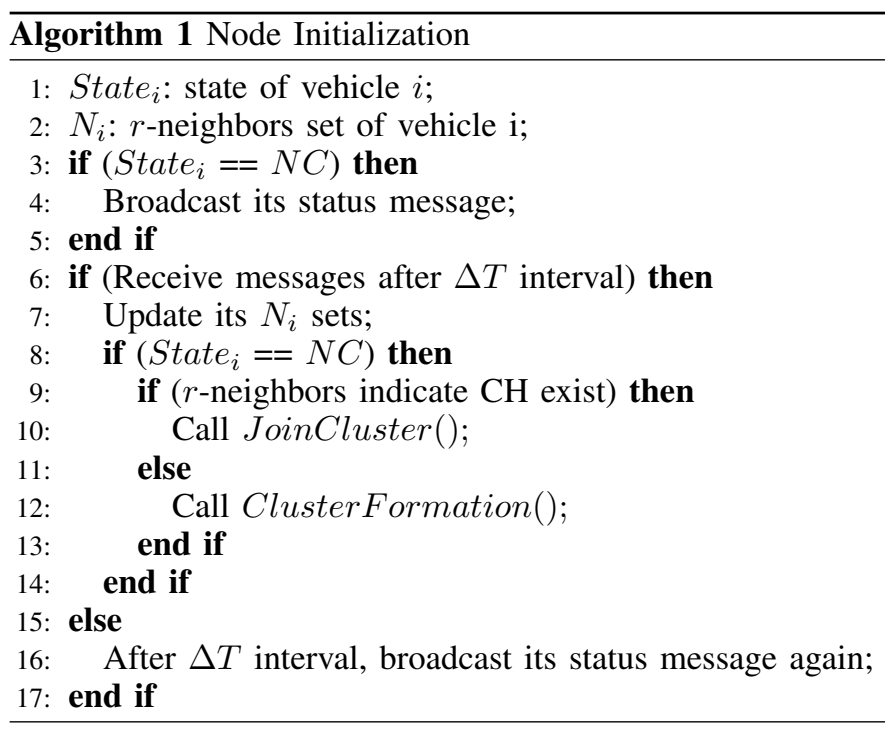

\section{B. Cluster Head selection phase}

In principle, $\mathrm{CH}$ is a coordinator with the task of coordination among cluster members and also responsible for management of cluster structure [20]. One of the most frequently used technique to increase cluster stability is $\mathrm{CH}$ duration. $\mathrm{CH}$ duration has impact direct relative with cluster stability. It means that select a more stable $\mathrm{CH}$ can be beneficial to keep cluster structure for long periods and stable cluster can reduce packet loss probability. Consequently, select a $\mathrm{CH}$ that can be stable for long period, is an important factor in the design of MOSIC. In proposed scheme, we defined a mobility measure, $M_{i}$, that each vehicle calculated it based on status messages which received in interval time $\Delta T$ from $r$-neighbors and each vehicle has greatest value of $M_{i}$, will be selected as $\mathrm{CH}$. Mobility measure calculated as follow:

$$
M_{i}= \begin{cases}\left|\frac{1}{M_{i}}\right| & , \bar{M}_{i} \neq 0 \\ 0 & , \bar{M}_{i}=0\end{cases}
$$

where $\bar{M}_{i}$ is average relative mobility. As mentioned in sec: III-D3, a vehicle with lowest value of $\bar{M}_{i}$ is more considered to be $\mathrm{CH}$, So, for simplicity calculations, the value 
of $\bar{M}_{i}$ will be reversed, because the lowest value becomes to the greatest value, And it's exactly what Formula 14 shows.

Once status message are received, the vehicle with highest $M_{i}$ among its $r$-neighbors will elect itself as $\mathrm{CH}$. Vehicle with highest $M_{i}$ will set its $C H I D$ field to its own $I D$ and send the status message to $r$-neighbors and subsequently all $r$-neighbors will join cluster (All $r$-neighbors sets their $C H I D$ field to vehicle's ID which selected as $\mathrm{CH}$ ). It should be noted that nodes with Non-Clustered state, can't participate in the election process and they must commence the initialization phase. 2.

The pseudo code of the $\mathrm{CH}$ selection shown in Algorithm

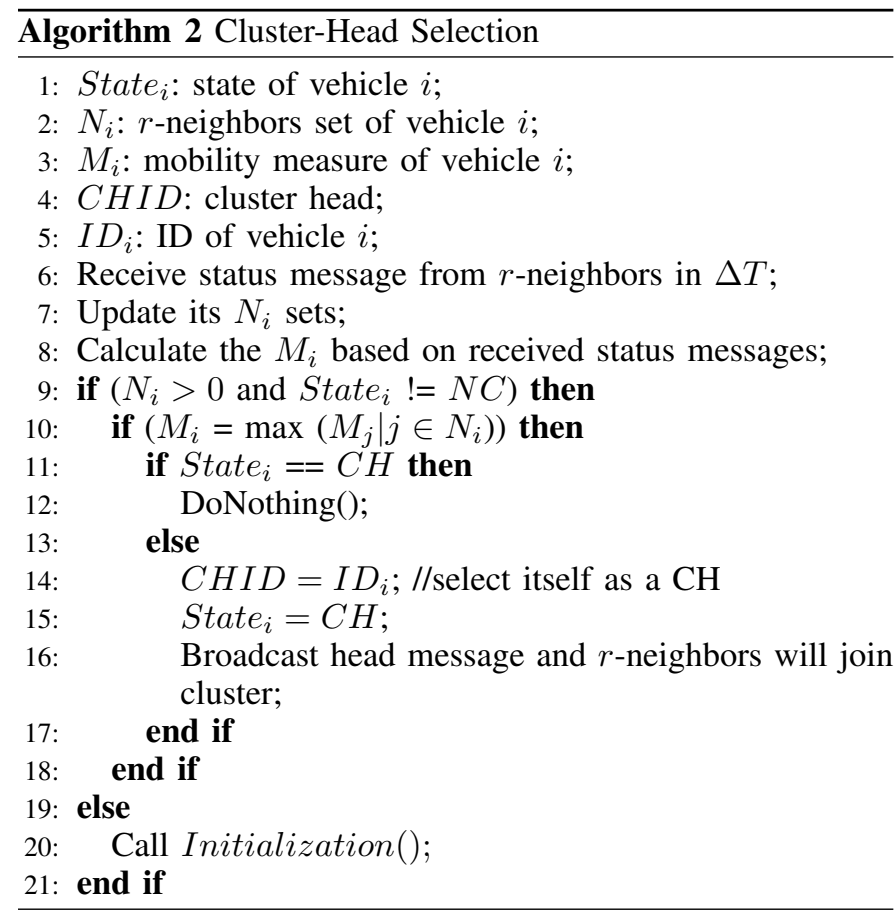

\section{Cluster formation phase}

The cluster formation phase is executed every time interval, $\Delta T$, with nodes in $\mathrm{NC}$ state that already before run the initialization phase and discover that there is no $\mathrm{CH}$ in vicinity. However, after initialization phase (which all NC state nodes broadcast its status message and receive reply messages), a vehicle whose speed is the slowest among all its $\mathrm{NC} r$ neighbors, start the cluster formation process. This vehicle is called cluster forming vehicle $(C F V)$. At the beginning of the process, $C F V$ select itself as a $C H$ and broadcast status message to $r$-neighbors. Thus vehicles upon receipt the status message, set its $C H I D$ field to $C F V$ 's ID and also update its state to $\mathrm{CM}$.

The pseudo code of the Cluster formation shown in Algorithm 3.

\section{Cluster maintenances phase}

The main aims of clusters maintenance phase is to maintain the cluster structure as stable as possible. Because of the dynamic nature of the VANET, joining and leaving the cluster happen frequently. However, there are three events that can affect on stability of a cluster include: Joining Cluster, Leaving Cluster and Cluster merging. In the following cluster maintenance procedure will be described.

1) Joining Cluster: When a NC (Non-Clustered) state vehicle approach a $\mathrm{CH}$ (comes within $\mathrm{CH}$ transmission range), then the vehicle and $\mathrm{CH}$ compare and check their relative velocity, $\bar{V}_{r e l_{i}}$, and if the velocity difference is within $\pm \Delta V_{t h}$, then the vehicle will join to the cluster and subsequently, $\mathrm{CH}$ add it to its members list. In some cases, a NC state's vehicle maybe comes in multiple $\mathrm{CHs}$ transmission range, $r$, then in this condition, vehicle join to cluster which has more nodal degree.

2) Leave Cluster: When a cluster-member moves out of the $\mathrm{CH}$ 's transmission range, $r$, it is not removed from the cluster members list maintained by the cluster-head immediately. In the other hand, if a $\mathrm{CM}$ does not receive the information broadcasted by the $\mathrm{CH}$ every $\Delta T$ interval, the state of this node changes from CM to TCM (Temporary Cluster Member). It does not leave the cluster immediately, because this disconnection maybe due to the weak quality of the wireless signals. If the temporary member receives the information broadcasted by the $\mathrm{CH}$ again in the coming $\mathrm{m}$ interval, the state of this node changes to $\mathrm{CM}$ again. But when a temporary member does not receive the $\mathrm{CH}$ information consecutively for $\mathrm{m}$ times, it means that the node moves out of the cluster range. Thus the state of that node changes to the NC. Meanwhile, the $\mathrm{CH}$ will delete this member from the members list. Then, the node can either join another cluster or form a new cluster.

3) Cluster Merging: Whenever two $\mathrm{CH}$ approach and come in each other transmission ranges, and they stay connected over a time period and also their relatively velocity is within the $\pm \Delta V_{t h}$, then the cluster merging process will commence. In this process, the $\mathrm{CH}$ with less nodal degree abandon their $\mathrm{CH}$ 's role and joins to the cluster with more nodal degree. The other members of the merged $\mathrm{CH}$ according to its condition can join another cluster or become a standalone member (NC).

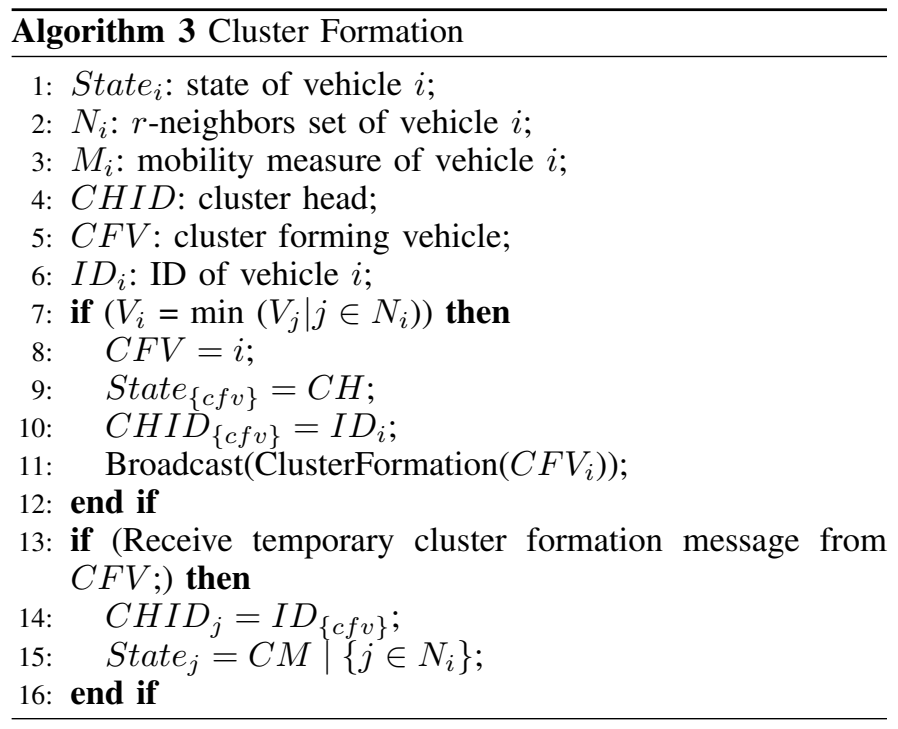


TABLE III: Simulation Parameters

\begin{tabular}{|l|l|}
\hline Parameter & Value \\
\hline Simulation Time & $300 \mathrm{~s}$ \\
Area Size & $1000 \mathrm{~m} \times 1000 \mathrm{~m}$ \\
Number of lans for each direction & 3 \\
Maximum Vehicle Velocity & $10-35 \mathrm{~m} / \mathrm{s}$ \\
Maximum Allowed Velocity & $55 \mathrm{~m} / \mathrm{s}$ \\
The threshold for stable $r$-neighbors & 10 \\
Number of vehicles & 100 \\
Interval Time & $1 \mathrm{~s}$ \\
Packet Type & UDP \\
Packet Sze & 100 Bytes \\
Transmission Range & $200 \mathrm{~m}$ \\
Chanel & IEEE-802.11 \\
Tuning parameter $(\alpha)$ & 0.85 \\
\hline
\end{tabular}

\section{Simulation and Performance Evaluation}

The aim of the simulation is to compare the performance of the our proposed mobility-aware single hop clustering scheme (MOSIC) to the previously proposed VANET clustering schemes. However, the performance of the clustering scheme is evaluated by using the metrics of cluster head duration, cluster member duration, cluster head change rate, the number of cluster and control overhead, which these performance metrics can demonstrate the stability of our clustering scheme [14], [1].

The MOSIC is implemented in NS-3 simulator at version 3.24.1 [18]. The simulation scenario is based on one directional highway segment of $1000 \mathrm{~m}$ in length and three lanes. The vehicles are injected into the road randomly. Maximum Velocity varies from 10 to $35 \mathrm{~m} / \mathrm{s}$ and the transmission range is $200 \mathrm{~m}$. The total simulation time is $600 \mathrm{~s}$. The clustering process start at the 300th second where all the vehicle are on the road. All of the performance metrics are evaluated for the remaining $300 \mathrm{~s}$. Also we consider that the maximum allowed velocity in the road is $55 \mathrm{~m} / \mathrm{s}$. The general and important simulation parameters are listed in Table III. Also we used Gauss-Markov mobility model, as temporary hybrid model beside vehicles mobility. In other words, we used GaussMarkov mobility (GMM) model as a prediction model for calculated next position of vehicles, which used in equation 10 . We set $\alpha$, Tuning parameter, to 0.85 , as shown in Table III.

\section{A. Cluster-Head Duration}

Cluster-Head duration refers to the interval during which the vehicle' state is in $\mathrm{CH}$ and remain in this state until its state changed into $\mathrm{CM}$ or $\mathrm{NC}$. The average $\mathrm{CH}$ duration is calculated by dividing the total $\mathrm{CH}$ duration with the total number of state changes from $\mathrm{CH}$ to $\mathrm{CM}$ or NC. Figure 3 illustrate the average $\mathrm{CH}$ duration of MOSIC and other clustering schemes for different maximum vehicle velocities. In Figure 3, the average $\mathrm{CH}$ duration decreases when the vehicle velocity increases. The reason for this is that when the vehicle velocity increase, the topology of network becomes more dynamic and eventually this makes it difficult for $\mathrm{CHs}$ to maintain a relatively stable condition with their neighbor vehicles for a long period. As shown in Figure 3, the MOSIC has better performance in term of $\mathrm{CH}$ duration against N-Hop [1], AMACAD [7], ASPIRE [23] and Lowest-ID [16] respectively.

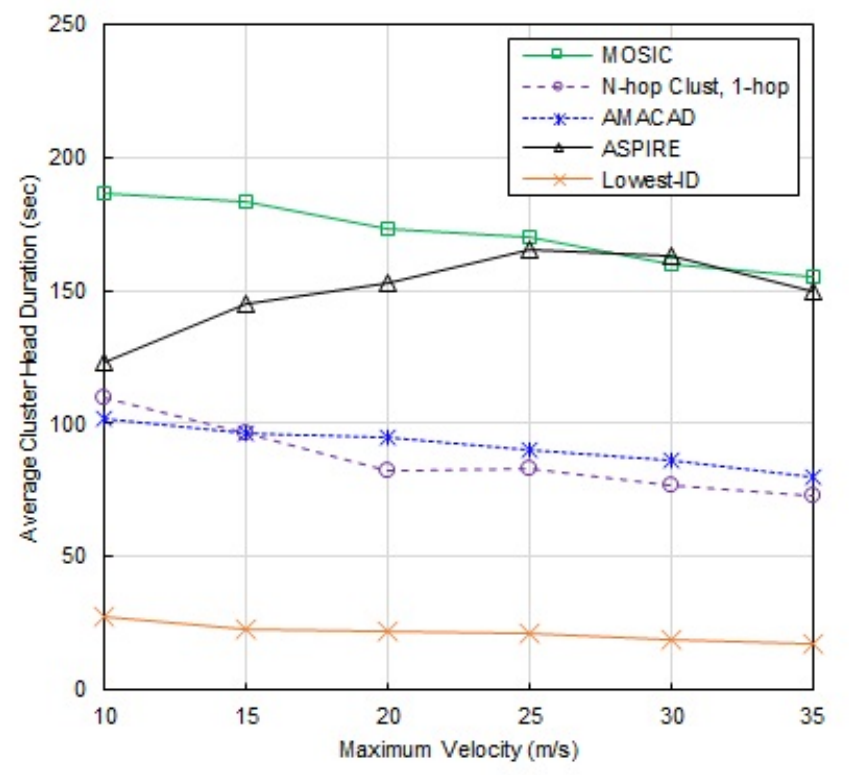

Fig. 3: Average CH Duration

\section{B. Cluster-Member Duration}

Cluster-Member duration is the interval from the time during which a vehicle joins a specified cluster to the time when it leaves the cluster. By dividing the total cluster member duration into total cluster member changes, average cluster member duration is calculated. Figure 4, shows the CM duration of MOSIC and other approaches for different maximum vehicle velocities. As shown in Figure 4, CM duration increases when vehicle velocity increases and it's because of the efficient cluster maintenance mechanism. The result which shown in Figure 4 indicate that the MOSIC CM duration is higher than N-Hop, AMACAD, ASPIRE and Lowest-ID respectively in most cases.

\section{Cluster-Head Change Rate}

Cluster-Head change number is the number of vehicles whose state changes from $\mathrm{CH}$ to $\mathrm{CM}$ or $\mathrm{NC}$ during a simulation process, and the rate of $\mathrm{CH}$ Change is defined as the changing per unit time. Figure 5, shows the $\mathrm{CH}$ change rate of MOSIC and other clustering schemes for different maximum vehicle velocities. A low $\mathrm{CH}$ change rate leads to a stable cluster structure. As shown in Figure 5, CH change rate increases when vehicle velocity increases. This is because of the dynamic nature of network. It means that with increasing velocity, it will be difficult for $\mathrm{CH}$ to keep efficient relatively stable with their CMs for a long period and maybe $\mathrm{CH}$ exited from cluster or in another condition, maybe $\mathrm{CH}$ into range of other $\mathrm{CHs}$ and merged with it and this situation effect of $\mathrm{CH}$ changes rate. 


\section{Number of Clusters}

In VANET, because of highly dynamic movement of vehicles, clusters are created and vanished frequently over time and it cause to increase clusters number and consequently, increase maintenance cost. A Few clusters can enhance efficiency and performance of VANETs. A clustering algorithm is suitable, if it could reduces the number of clusters in system. This suitability achieved by create a relatively metric which sustain the current cluster scheme stable as much as possible. Figure 6 demonstrate the number of clusters under different transmission ranges and velocity scenarios. As shown in Figure 6, With increasing velocity the changes in the number of clusters is minimally and it because of good relative mobility metric which utilized in our scheme.

\section{E. Average Control Message Overhead}

All clustering schemes incur some additional control overhead to form and maintain their cluster structures and most of this overhead related to cluster formation and $\mathrm{CH}$ selection. So, in this paper we consider the overhead of the cluster formation and cluster head selection as the control message overhead. However, the average control message overhead is the count of total control message received by each vehicle in the network at cluster formation and $\mathrm{CH}$ selection procedures. Figure 7 shows the average control message overhead of MOSIC, NHop, AMACAD and ASPIRE at different velocities. Compared with above-mentioned clustering algorithm, MOSIC performs better in terms of control overhead. In MOSIC, each vehicle creates a control message during every channel interval and broadcast it to its single-hop neighbors to calculate the relative mobility between vehicle and its neighbors. This condition is equal to all above-mentioned clustering algorithms. But because of hight stability of cluster structure in MOSIC, with more $\mathrm{CH}$ duration and low $\mathrm{CH}$ change rate, the control message to reestablish the clusters structure and $\mathrm{CH}$ selection will be reduced and consequently the control overhead will be decreased.

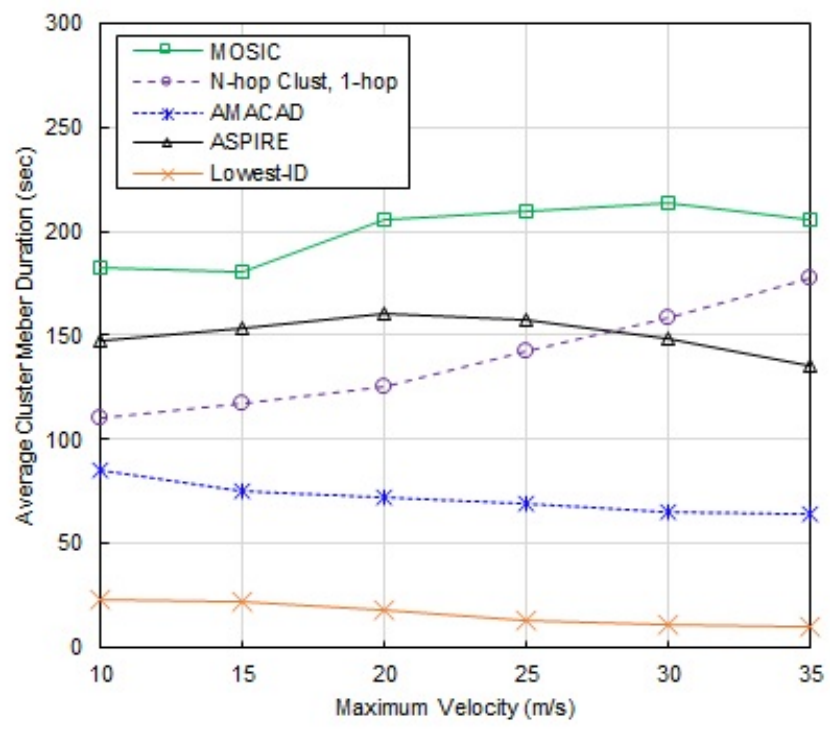

Fig. 4: Average CM Duration

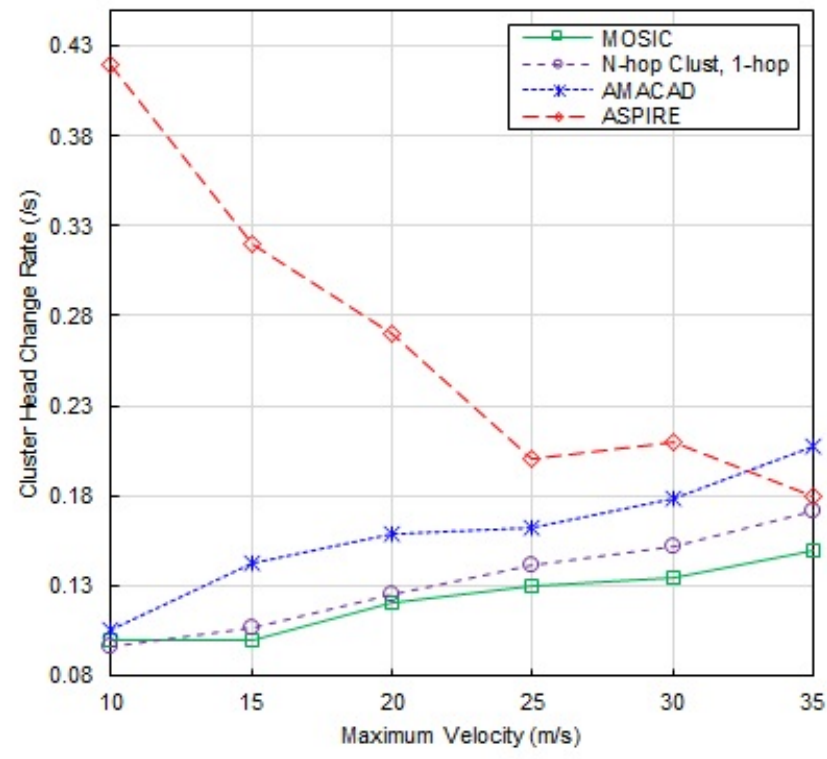

Fig. 5: Average CH Changes Rate

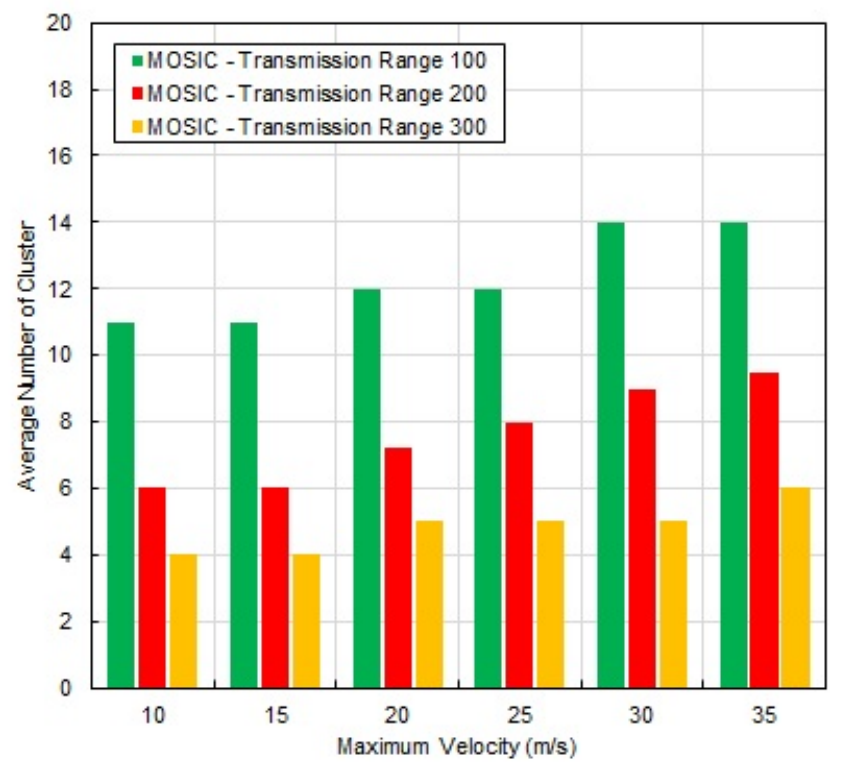

Fig. 6: Average Number of clusters

\section{CONCLUSION}

Clustering mechanism is one of existence organizing mechanism which designed to adapt to the VANET environment. In this study, a mobility-aware and single-hop clustering scheme (MOSIC) was proposed. The MOSIC is based on the changes in the relative mobility of the vehicles, which is calculated by finding the average of the relative velocity, the nodal degree and relative distance of all the same direction neighbors. It used Gauss-Markov mobility model to predict the vehicle next location and based on the vehicle's location and its predicted location, relative distance will be calculated and consequently 
relative mobility can be obtained. The MOSIC simulated on NS-3 and its performance compare to some clustering approach. Simulation indicate that the clustering of MOSIC outperforms than N-Hop, AMACAD, ASPIRE and Lowest-ID clustering in terms of $\mathrm{CH}$ duration, $\mathrm{CM}$ duration, $\mathrm{CH}$ change rate metrics and Control Message Overhead at various vehicle velocity scenarios. As future work, we aim to investigate the use of MOSIC in urban traffic scenarios and design the efficient routing protocol based on this scheme.

\section{REFERENCES}

[1] Zhang, Z., Boukerche, A. and Pazzi, R.: "A novel multi-hop clustering scheme for vehicular ad-hoc networks", Proceedings of the 9th ACM international symposium on Mobility management and wireless access, New York (2011), 19-26.

[2] Bali, R., Kumar, N. and Rodrigues, J.: "Clustering in vehicular ad hoc networks: Taxonomy, challenges and solutions"; Vehicular Communications, 1, 3 (2014), 134-152.

[3] Liang, B. and Haas, Z.: "Predictive distance-based mobility management for PCS networks"; IEEE INFOCOM '99. Conference on Computer Communications. Proceedings. Eighteenth Annual Joint Conference of the IEEE Computer and Communications Societies. New York (1999), 1377-1384.

[4] Basu, P., Khan, N. and Little, T. (n.d.).: "A mobility based metric for clustering in mobile ad hoc networks". Proceedings 21st International Conference on Distributed Computing Systems Workshops, Mesa, AZ (2001), 413-418.

[5] Souza, E., Nikolaidis, I. and Gburzynski, P.: "A New Aggregate Local Mobility (ALM) Clustering Algorithm for VANETs“. 2010 IEEE International Conference on Communications, Cape Town, South Africa (2010), 1-5.

[6] Lin, C. and Gerla, M.: "Adaptive clustering for mobile wireless networks“. IEEE J. Select. Areas Commun., 15, 7 (97), 1265-1275.

[7] Selvam, R. P., Palanisamy, V.: "Stable and flexible weight based clustering algorithm in mobile ad hoc networks", international Journal on Computer Science and information Technology, 2, 2 (2011), 824-828.

[8] Yu, J. and Chong, P.: "3hBAC (3-hop between adjacent clusterheads): a novel non-overlapping clustering algorithm for mobile ad hoc networks",

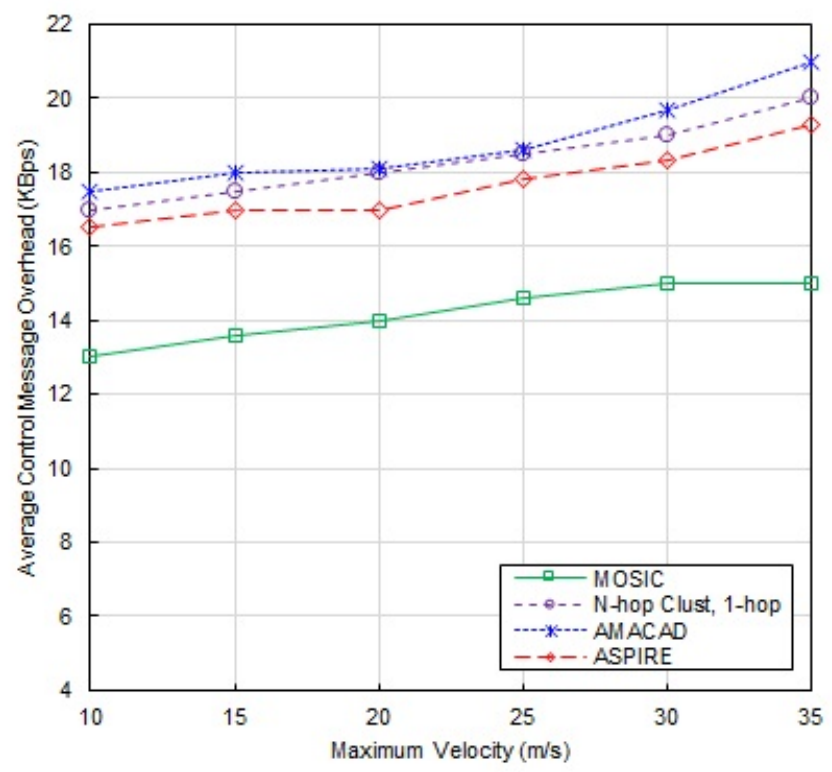

Fig. 7: Average Control Message Overhead
IEEE Pacific Rim Conference on Communications Computers and Signal Processing, PACRIM (2003), 318-321.

[9] Yadav, N.S., Deosarkar, B.P., Yadov, R.P.: "A low control overhead cluster maintance scheme for mobile ad hoc networks", International Journal of recent trends in engineering, 1, 1 (2009), 1-9.

[10] Bentaleb A., Boubetra A., Harous S.: "Survey of clustering schemes in mobile ad hoc networks", Communication Networks, 5, 2 (2013), 8-14.

[11] Ni, M., Zhong, Z. and Zhao, D.: "MPBC: A Mobility Prediction-Based Clustering Scheme for Ad Hoc Networks“. IEEE Trans. Veh. Technol., 60, 9 (2011), 4549-4559.

[12] Xu, Y., Bien, S., Mori, Y., Heidemann, J., Estrin, D.: “Topology control protocols to conserve energy in wireless ad hoc networks", center for Embedded Network Sensing, 2003, 1-18.

[13] Eichler, S.: "Performance Evaluation of the IEEE 802.11p WAVE Communication Standard", IEEE 66th Vehicular Technology Conference, Baltimore (2007), 2199-2203.

[14] Shea, C., Hassanabadim B., Valaee, S.: "Mobility-Based Clustering in VANETs Using Affinity Propagation“, IEEE Global Telecommunications Conference, Honolulu (2009), 1-6.

[15] Hafeez, K.A., Zhao, L., Mark J.W., Shen, X., Niu, Z.: "Distributed Multichannel and Mobility-Aware Cluster-Based MAC Protocol for Vehicular Ad Hoc Networks", IEEE Transactions on Vehicular Technology, 62, 8 (2013), 3886-3902.

[16] Lin, C. R., Gerla, M.: "Adaptive clustering for mobile wireless networks". IEEE Journal on Selected Areas in Communications, 15, 7 (1997), 1265-1275.

[17] Morales, M., Hong, C. and Bang, Y.: "An Adaptable Mobility-Aware Clustering Algorithm in vehicular networks". 13th Asia-Pacific Network Operations and Management Symposium, Taipei (2011), 1-6.

[18] Network simulator 3 (ns-3), Discrete Event Network Simulator, Sep. 2015. [Online]. Available: http://www.nsnam.org.

[19] Mohammad, S. and Michele, C.: "Using traffic flow for cluster formation in vehicular ad-hoc networks". IEEE Local Computer Network Conference, Denver (2010), 631-636.

[20] Su, H. and Zhang, X.: "Clustering-Based Multichannel MAC Protocols for QoS Provisionings Over Vehicular Ad Hoc Networks“. IEEE Transactions on Vehicular Technology, 56, 6 (2007), 3309-3323.

[21] Gupta, N., Prakash, A., Tripathi, R.: "Medium access control protocols for safety applications in Vehicular Ad-Hoc Network: A classification and comprehensive survey“. Vehicular Communications, 2, 4 (2007), 223237.

[22] Cheng, H. T., Shan, H., Zhuang, W.: "Infotainment and road safety service support in vehicular networking: From a communication perspective“. Mechanical Systems and Signal Processing, 25, 6 (2011), 20202038.

[23] A. Koulakezian. (2011, August). ASPIRE: Adaptive Service Provider Infrastructure for VANETs. Master thesis at The University of Toronto. [Online]. Available: http://hdl.handle.net/1807/29581. 